\title{
An Improved Multi-threading Data Acquisition System of Acoustic Signal
}

\author{
Ma Qingfeng, Liu Hui \\ Shandong Provincial Key Laboratory of Ocean \\ Environment Monitoring Technology \\ Shandong Academy of Sciences Institute of \\ Oceanographic Instrumentation \\ Qingdao, China,266001
}

\author{
Cui Hao, Xiang Xu \\ Shandong Provincial Key Laboratory of Ocean \\ Environment Monitoring Technology \\ Shandong Academy of Sciences Institute of \\ Oceanographic Instrumentation \\ Qingdao, China,266001
}

\begin{abstract}
Some problems such as long processing cycle and false alarm exist in traditional target detecting. An improved multi-threading data acquisition system is introduced in this paper for the solution. In this system independent collectors cooperation mode is injected, which could acquire acoustic signal in multi-threads way. It could increase the real-time, reliability and decrease the false alarm. The system could be installed into fixed buoys, drifting buoys or subsurface buoys to acquire, analyze acoustic signals and make early-warning. It exceeds former systems on extending the detecting range and accuracy through independent collectors' cooperation.
\end{abstract} Buоy

Keywords- Data acquisition, Multi-threads, Subsurface buoy,

\section{INTRODUCTION}

The direction, positioning, and self-identification of target is automatically carried out through the radiated submarine noise, which effectively results in the conservation of physical resource. The vector sensor is a perfect choice because the certain spatial gain can be gained in the low-frequency, small-scale formation, and at the same time, the accurate underwater target position information is also got. Additionally, a single acoustic vector sensor can provide the orientation information of sound field.

In 1932, Olson presented a method to test sound intensity: to multiply sound pressure and vibration velocity acquired and then get the integral [1]. But it apparently worked only under idealized conditions.

Recently, vector hydrophone is widely used to detect and trace target by the energy information of sound field and the orientation of target, which could be calculated by acquiring sound pressure and vibration velocity of three orthogonal directions.

This paper introduces the multi-threads data acquisition system of underwater acoustic signal with high real-time and reliability. The intellectualized system could acquire and analyze acoustic signals and make early warning. It could be installed into fixed buoys, drifting buoys and subsurface buoys.

\section{RESEARCH CONTENT}

The tranditional target detection systems have some problems, eg. long data processing cycle, unreliability, false alarm and so on. In order to solve these issues, we introduce a novel multi-threads data acquisition system of underwater acoustic signal, which could locate target by multi-systems working collaboratively using bearings-only location method.

\section{A. collaborative work of multi-threads}

In order to improve the real-time and reliability of the system, we use one controller and three data collectors to acquire sound pressure and vibration velocity of vector hydrophone time-sharingly, and cumulate energy, analyze the data and send results to shore station separately. If one target is detected in the sound field, the system would report target's location every one third cycle. With multi-systems working collaboratively, we could locate and trace the target timely. Data collectors have the same priority, which could make one to be a hot backup of others. Controller could shield failure collectors to keep the whole system working well. System structure is shown in Figurel.

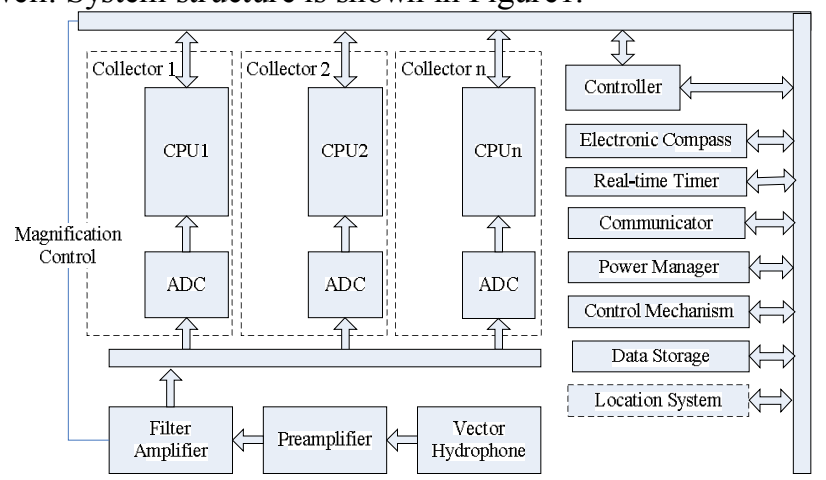

Figure 1. System structure

\section{1)Controller}

The controller consists of MSP430F149 as processor with bus, serial interface and extended device and circuits. It is mainly used to control and harmonize the collects' order and communication between shore station and system. There is no limits to thread in theory, but by considering complexity of equipment and interval into account, we use three data collectors. Multi-threads structure is shown in Figure2. 


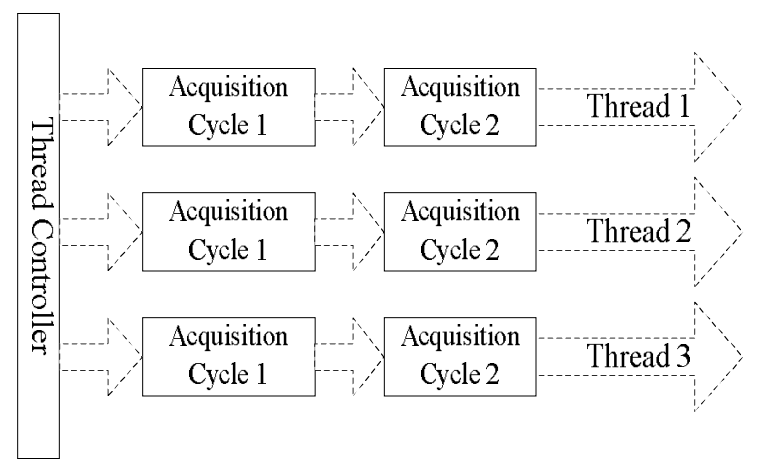

Figure 2. Multi-threads structure

Controller devides one data processing cycle into three stages. First of all, turn on collectors in each stage on time, check status of collectors, set up a management table of threads, and then eliminate abnormal threads. Second, specify a main thread with high priority in the management table. The main thread could control the magnification of filter amplifier and send messages to shore station. Third, controller allows threads working independently, wait for the data from threads, and send the result to shore station.

2) Collector

ATMEGA2560 is mainly taken in collector with AD7859, RAM, CF card and other devices as peripherals. It has functions of self-calibration filter amplifier, selfchecking, energy accumulation, spectrum analyzing and location estimation.

The sensor outputs signal by 4 channels: one of sound pressure $(\mathrm{P})$ and three of vibration vibration $(\mathrm{X}, \mathrm{Y}, \mathrm{Z})$. The 4 signals are sent into collector after preamplifier and filter amplifier adjusting. When self-checking, collector samples 8000 discrete data points from each channel, and then determines the work state by analyzing. The judgment rule is that if data mean matches bias, channel works well; otherwise, channel fails, then the result is send to controller. The workflow of self-checking is shown in Figure3.

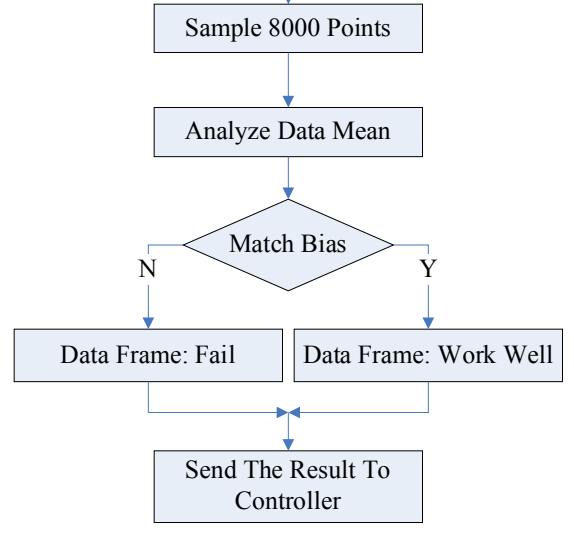

Figure 3. Workflow of system self-checking

Adaptive adjusting magnification could keep useful signal from background noise, if signal saturates. It could also prevent sensitivity decline if magnification is too low, and ensure that the data contains accurate information of sound field. The judgment rule is that: after analyzing 8000 sampling points, if amplitudes of over $1 / 5$ of the data achieve $90 \%$ of the whole range $(2.5 \mathrm{~V})$, so the magnification of filter amplifier is too high. And if amplitudes of over $2 / 3$ of the data are below $20 \%$ of the whole range $(2.5 \mathrm{~V})$, the magnification of filter amplifier is too low. The workflow of adaptive adjusting magnification is shown in Figure4.

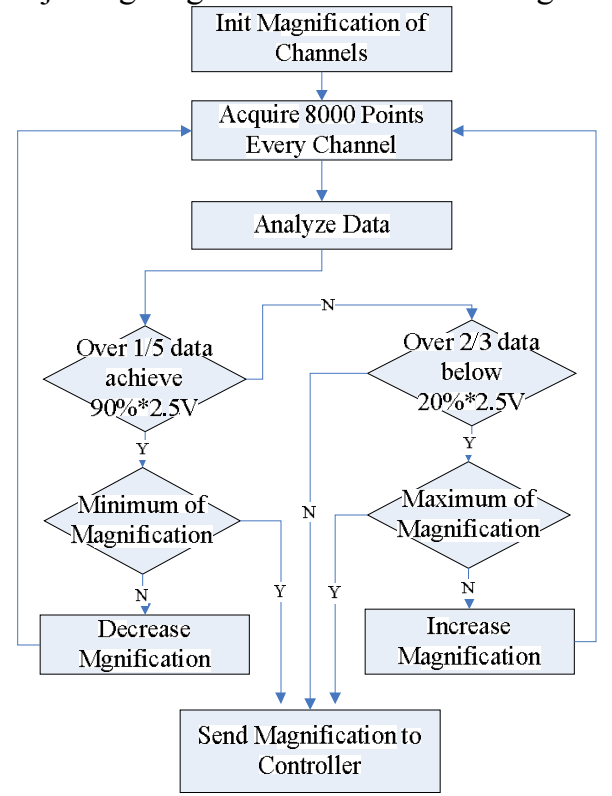

Figure 4. Workflow of adaptive adjusting magnification

The maximum direction-finding algorithm is adopted according to the azimuth estimation. This algorithm is usually used and effective in the sonar systems. the output signal of vector hydrophone changes with the azimuth modification of target, therefore, the measurment of target position can be got through the signal got by the energy converters and the arrays. The pressure $\mathrm{P}$ and the velocity $\mathrm{X}$, $\mathrm{Y}$, and $\mathrm{Z}$ is used in this algorithm, and the angle spectrum is got through the cross spectrum or the cross-correlation, finally, the target direction is obtained according to the angle corresponding to the maximum sound intensity. This method is not directly measured about the phase difference, therefore, it is an indirect measurement algorithm. It takes advantage of the direction of sound system in order to the target orientation.

The specific azimuth estimation is as follow:

Calculate the Cross-spectrum with sound pressure and vibration velocities along $\mathrm{X}$-axis and $\mathrm{Y}$-axis, get the target quadrant according to the above result, and estimate the azimuth by anti-arctangent trigonometric function, as is shown in formula $2.1[2]$.

$$
\alpha=\operatorname{arctg}\left(\frac{I_{y}}{I_{x}}\right)
$$

$I_{x}$ is the Cross-spectrum of sound pressure and vibration velocities along $\mathrm{X}$-axis $\left(\bar{V}_{x}\right)$. 
$I_{y}$ is the Cross-spectrum of sound pressure and vibration velocities along Y-axis $\left(\overline{V_{y}}\right)$.

3)Signal Conditioning Circuit

Signal coordinating circuit includes preamplifier and filter amplifier, which includes band-pass, changeable magnification and other functions. This is not emphasis of the paper, so it will not be discussed in detail.

4)Hydrophone

This paper introduces very low frequency (VLF) composite hydrophone, including three groups of vibration velocity sensors and one group of sound pressure sensors. The sensitivity and impedance consistency of vibration velocity sensors along $\mathrm{X}$-axis and $\mathrm{Y}$-axis should ensure the same. Uwhole

\section{B. collaborative work of multi-systems}

Nowadays, the detection system could not locate the target directly. In order to improve the efficient and accuracy, muti-systems should be laid in certain area of sea, forming a detection array. Shore station receives data from detection array, estimates the target location using the method of bearings-only location [3], and traces the target on chart.

The method of bearings-only location uses two or more location equipment to locate target. We could get the target azimuth and the distance between equipments, and use the trigonometric equation to locate the target. Because the distance between multi-systems and target is far more than the depth of target in offing, we could simplify the computation by considering the multi-systems and the target on the same level. The method of bearings-only location is shown in Figure5. A and B are detection systems. $G$ is the target. we have known the distance between $\mathrm{A}$ and $\mathrm{B}$, the target azimuth $\alpha 1$ computed by A and the target azimuth $\alpha 2$ by $\mathrm{B}$. Then the distance between $\mathrm{A}$ and $\mathrm{G}$ and the distance between $B$ and $G$ could be computed by trigonometric equation. Using the result we could get the location of $\mathrm{G}$.

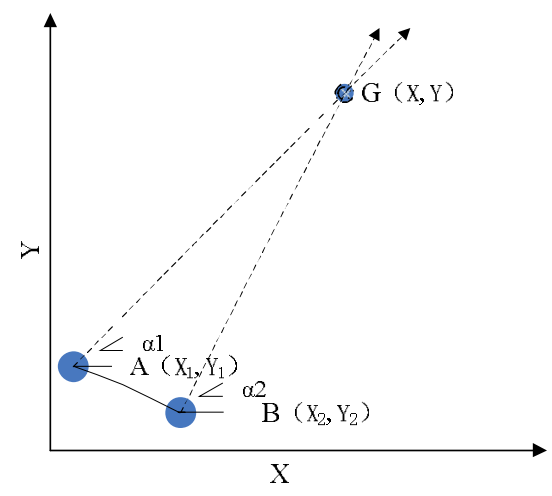

Figure 5. The method of bearings-only location

As is shown in Figure5, let the observation station coordinates of double subsurface buoys be A ( $\mathrm{x} 1$, , y1 ), B ( $\mathrm{x} 2$, y2 ), let target coordinates be G ( $x, y$ ), and let $\alpha 1$ and $\alpha 2$ be the measurements of corresponding Angle of A and B.

The matrix equation form positioning:

$$
\left[\begin{array}{l}
1-\tan \alpha_{1} \\
1-\tan \alpha_{2}
\end{array}\right]\left[\begin{array}{l}
y \\
x
\end{array}\right]=\left[\begin{array}{l}
y_{1}-x_{1} \tan \alpha_{1} \\
y_{2}-x_{2} \tan \alpha_{2}
\end{array}\right]
$$

From 2-2, the positioning error expressions can be drawn through Differentiating:

$$
\begin{gathered}
d X=C^{-1}\left(d V-d X_{s}\right) \\
d X=\left[\begin{array}{l}
d y \\
d x
\end{array}\right], d V=\left[\begin{array}{l}
d \alpha_{1} \\
d \alpha_{2}
\end{array}\right] \\
C=\left[\begin{array}{ll}
\frac{-\sin ^{2} \alpha_{1}}{y-y_{1}} & \frac{\cos ^{2} \alpha_{1}}{x-x_{1}} \\
\frac{-\sin ^{2} \alpha_{1}}{y-y_{2}} & \frac{-\cos ^{2} \alpha_{2}}{x-x_{2}}
\end{array}\right) \\
d X_{s}=\left[\begin{array}{ll}
\frac{\sin ^{2} \alpha_{1}}{y-y_{1}} d x_{1} & -\frac{\cos ^{2} \alpha_{1}}{x-x_{1}} d y_{1} \\
\frac{\sin ^{2} \alpha_{1}}{y-y_{2}} d x_{2}-\frac{\cos ^{2} \alpha_{2}}{x-x_{2}} d y_{2}
\end{array}\right]
\end{gathered}
$$

$d V$ is Measurement error. $C$ is the transition matrix. $d X_{s}$ is the hail error.

The location error covariance matrix [4]

$$
\begin{aligned}
& P_{d x}=E\left[d X \bullet d X^{T}\right]= \\
& C^{-1}\left\{E\left[d V \bullet d V^{T}\right]+E\left[d X_{s} \bullet d X_{s}^{T}\right]\right\} C^{T}
\end{aligned}
$$

Geometrica 1 dilu tion of precision - GDOP ${ }^{[5]}$ can be used to describe the positioning error in $\mathrm{X}-\mathrm{Y}$ plane distribution.

In order to facilitate analysis and discussion, where the system baseline are selected in the $\mathrm{X}$-axis coordinates, the target can be got in the plane $(x, y)$. The GDOP mathematical expression [5]:

$$
G D O P=\frac{r_{1} r_{2}}{|y| d}\left[r_{1}^{2} \sigma_{\alpha 1}^{2}+r_{2}^{2} \sigma_{\alpha 2}^{2}+2 \sigma_{s}^{2}\right]^{\frac{1}{2}}
$$

Here, $d$ is the length of baseline; $r_{1}$ and $r_{2}$ is the distance of target-subsurface buoy; $\sigma_{\alpha 1}$ and $\sigma_{\alpha 2}$ are the standard deviation of azimuth measurement; $\sigma_{\mathrm{s}}$ is the standard deviation of hail measurement.

Previous work show that if detection area is suitable, it is easy to get more accurate target location by augmenting the distance between A and B. Direction error has great influence of location precision. Small direction error could lead a large error of location, and the minimum of direction error is existing from mathematics. This error is $\sqrt{2}$ times the hail one[4].

Under current condition of technology, in order to get more accurate result, it is needed to lay numerous detection systems in the same sound field. Because the errors in target azimuth and coordinate of system, the location would be located in a small area. But there would reduce cost in antisubmarine warfare. Multi-systems location is shown in Figure6. $\alpha 1, \alpha 2$ and $\alpha 3$ are computed by the detection system of $\mathrm{A}, \mathrm{B}$ and $\mathrm{C}$. 


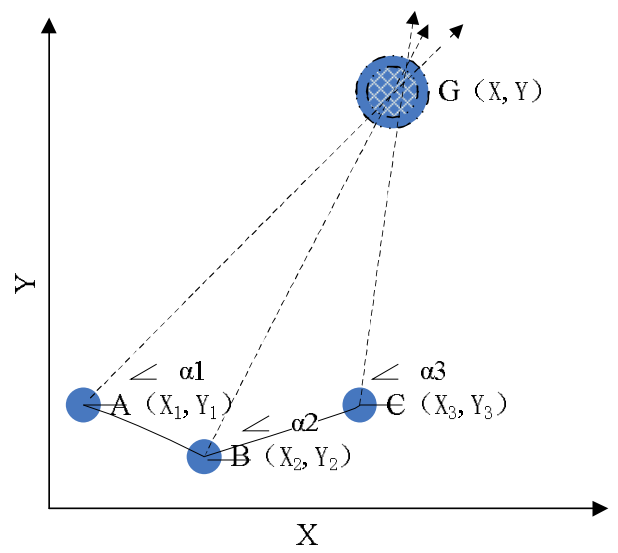

Figure 6. Multi-systems location

\section{CONCLUSION}

This paper introduces an improved multi-threads data acquisition system of underwater acoustic signal. The research shows that the system has higher reliability and lower probability on false alarm and omission alarm. And the target could be located effectively by multi-systems, which would be useful in national defense.

\section{ACKNOWLEDGMENT}

This paper was supported by the National Natural Science Foundation of China under Grant 40806044 and Supported by the Qingdao Science and Technology Planning Project Fund, China under Grant 10-3-4-9-2-jch, and supported by Research Fund for the Doctoral Program of the Shandong Academy of Sciences under Grant Y09-2.

\section{REFERENCES}

[1] H. F. Olson, System Responsive to The Energy Flow in Sound Waves [P], U. S. Patent 1892644, 1932.

[2] Pan Rui-yun, Design and Implement of Data Acquisition System of Underwater Acoustic Signal [D], MS Thesis of HarBin Engineering University, 2009 (In Chinese).

[3] GAVISH M, Analysis of bearing-only target location algorithms [J], IEEE Transactions on Aerospace and Electronic systems, 1992.

[4] Zhang yu, Sun Da-jun, Lv Yun-fei, Lan Hua-lin, Analysis of Location Precision when Using a Dual Subsurface-buoy System with a Vector Hydrophone [J], Journal of Harbin Engineering University, 2010.7 (In Chinese).

[5] Sun Zhong-kang, Zhou Yi-yu, He Li-xing. Active and passive single-multi base location technology [M]. Beijing: National Defence Industry Press, 1996: 37-50. 